\title{
FABRICATION AND MEASUREMENT OF AN IC-COMPATIBLE GHZ-RANGE PIEZOELECTRIC LONGITUDINAL BAR RESONATOR
}

\author{
D. J. D. Carter, J. Kang, D. White, and A. E. Duwel \\ The Charles Stark Draper Laboratory \\ Cambridge, MA 02139
}

\begin{abstract}
We report on the fabrication and measurement of a piezoelectrically-actuated, longitudinal-mode acoustic MEMS resonator designed to operate in air with high $Q$ and low impedance in the $\sim 50 \mathrm{MHz}-2 \mathrm{GHz}$ frequency range. Since the longitudinal-mode frequency is set lithographically by the lateral bar dimensions, a wide range of resonator frequencies can be created on a single chip. Device processing is IC compatible, offering the potential for integration in transceiver applications.

This first set of fabricated devices had resonator lengths ranging from 3 to 30 microns, corresponding to frequencies from $100 \mathrm{MHz}$ to $1.3 \mathrm{GHz}$, on a single wafer. We discuss the fabrication process for these aluminum nitride (AIN) devices and present measurements on resonators with frequencies ranging from $\sim 100-700 \mathrm{MHz}$.
\end{abstract}

\section{INTRODUCTION}

Radio-frequency transceivers used today rely on off-chip components for highly frequency selective resonant elements. The ability to integrate extremely small, high quality factor (Q) resonators will significantly impact size and performance in existing transceivers [1]. In addition, new system architectures will take advantage of ultra-dense arrays of resonators to make chipscale filter banks and frequency synthesizers, enabling a new generation of small, universal transceivers.

Towards these goals, relevant performance metrics of the resonators include small size, high $\mathrm{Q}$, frequency scalability over 0.3-3 GHz, RF integrated circuit process compatibility, RF impedance compatibility, linearity, and power handling. On all fronts, the thin-film resonator (TFR) technology, which uses piezoelectric actuation, is particularly advanced. Resonators at 0.8-3 GHz with Q's ranging from 450-2000 and impedance levels in the range of $50 \mathrm{Ohms}$ are reported $[\mathbf{2 , 3 , 4}$. Quartz resonators have been demonstrated with Q's above 13,000 at $590 \mathrm{MHz}$ [5]. Several capacitively actuated resonator designs that are easily scalable have also demonstrated impressive Q's. In [6], a diskmode resonator at $1.14 \mathrm{GHz}$ produced $\mathrm{Q}=1500$ in air. Capacitively actuated designs are challenged however by high impedance levels (in the range of $10 \mathrm{k} \Omega$ ) and process compatibility.

The Draper piezoelectric longitudinal bar (L-Bar) resonator design strategically addresses key requirements for RF applications. In the following sections, we review the resonator design and several of its merits. The fabrication process is next described in detail. Finally, we present results from initial device measurements.

\section{RESONATOR DESIGN}

The L-Bar resonator physically resembles a suspended capacitor. Figure 1 shows a solid model of the center bar suspended above a silicon substrate via tethers. The bar is metalized on top and bottom. These electrodes apply a field through the thickness of the AlN. At most frequencies, the device response is that of a capacitor. At the mechanical resonance, however, the electrical impedance drops by orders of magnitude, depending on the device Q.

The choice of a piezoelectrically actuated, in-plane longitudinal mode MEMS device is unique and the design addresses important application metrics. The frequencies of inplane longitudinal modes are lithographically scalable over $\sim 50 \mathrm{MHz}-2 \mathrm{GHz}$, allowing arrays on a single chip. These modes are also robust to package (thermal) stress. The piezoelectric actuation allows for impedance matching. The suspended bar structure and materials choices enable extremely high Q designs. In addition, the use of aluminum nitride (AIN) as a structural and transduction material has multiple advantages. AlN can be deposited at temperatures well below $400^{\circ} \mathrm{C}$ for process compatibility, it has a low dielectric loss tangent, the dielectric breakdown is $\sim 10^{6} \mathrm{~V} / \mathrm{cm}$ (allowing for high power handling), and the response is extremely linear. Finally, the electrode geometry enables a self-aligned, manufacturable process and minimizes spurious modes so that additional mode selection filters are not necessary.

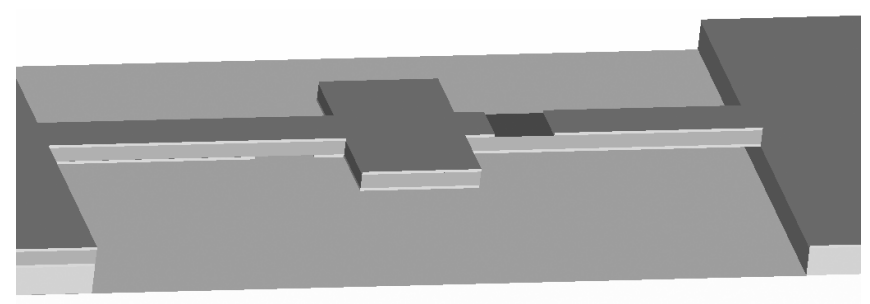

Figure 1. Schematic of the L-bar device. The suspended resonator is made of piezoelectric aluminum nitride sandwiched between metal layers. The resonant structure is suspended over a gap by tethers. Nominal dimensions for an $800 \mathrm{MHz}$ resonator are $5 \mathrm{um}$ long $x 3$ um wide with a 1.5 um wide tether.

\section{PROCESS OVERVIEW}

Figure 2 shows an overview of the process flow for the resonator. The starting point is the following materials stack on a silicon wafer: a release layer $(0.5-1 \mu \mathrm{m}$ thick $)$ of thermallygrown $\mathrm{SiO}_{2}$, a thin $(\sim 200 \AA)$, "seed" layer of AlN, $1500 \AA$ of Mo, and $5000 \AA$ of polycrystalline, c-axis-oriented, piezoelectric AlN. Approximately $2000 \AA$ of $\mathrm{Ni}$ is then patterned by evaporation and liftoff.

The patterned nickel serves as an etch mask for wet etching of AlN, which serves as an etch mask for RIE of the Mo bottom metal and a further wet etch of the seed AlN. At this point the mechanical form of the resonator, support tethers, and probe pads has been defined into the stack. Figure 3 shows a scanningelectron micrograph of an etched stack test structure. 


\section{Pattern top Ni by liftoff}

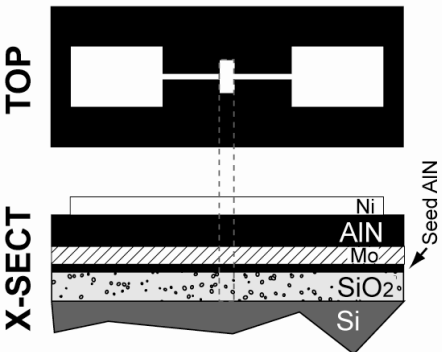

3. Pattern electrical circuit

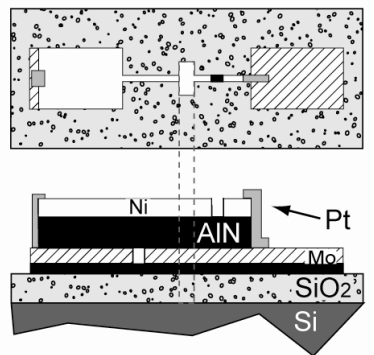

\section{Etch AIN/Mo/AIN stack}

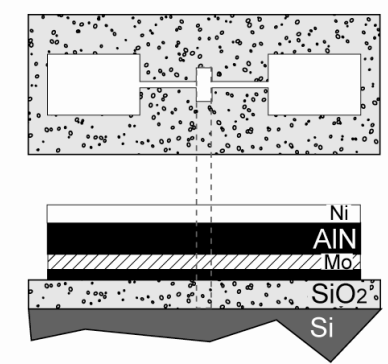

4. Release

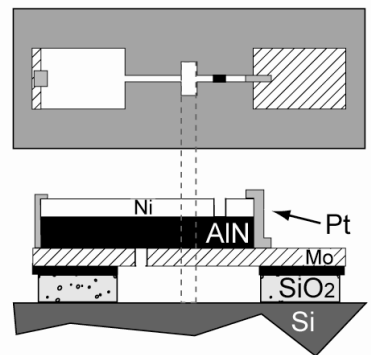

Figure 2. Schematic of L-bar process flow. Mechanical form of resonator, including critical alignments, is defined in first mask level. Each layer in the device film stack serves as a self-aligned etch mask for subsequent layers. Several etch steps pattern the electrical circuit. The release etch is self aligned.

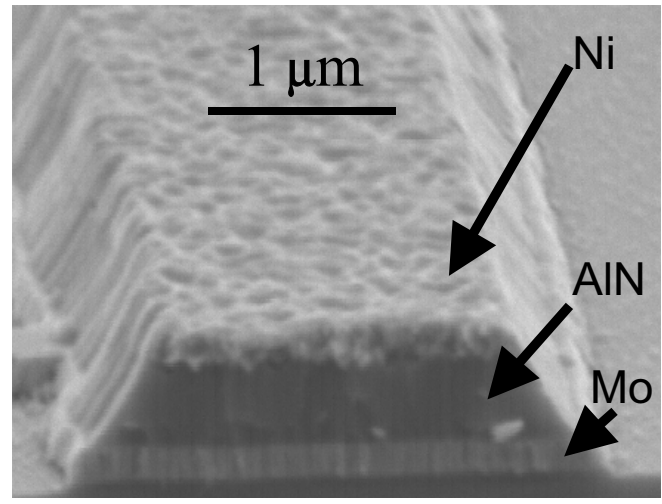

Figure 3. Scanning-electron micrograph of etched stack structure. Nickel, patterned by liftoff, serves as an etch mask for the underlying AlN, which is wet etched in AZ400k photoresist developer. The Ni and AlN serve as etch masks for RIE of the molybdenum bottom metal in CF4 plasma.

The next sequence of steps forms the proper electrical circuit. To minimize parasitic capacitances (i.e. top/bottom metal overlap other than on the resonator), breaks are etched into the tether Mo and $\mathrm{Ni}$ on opposite sides of the resonator. Then the Ni and AlN are removed by wet etching on the probe pad opposite the bottom metal break to allow electrical access to the Mo for testing. To complete the electrical circuit, isolated top and bottom metal islands on the tethers are shorted to appropriate pads by liftoff of a platinum "patch."

The device is released by etching the underlying $\mathrm{SiO}_{2}$ in BHF. Figure 4 shows scanning-electron micrographs of two released resonator devices.
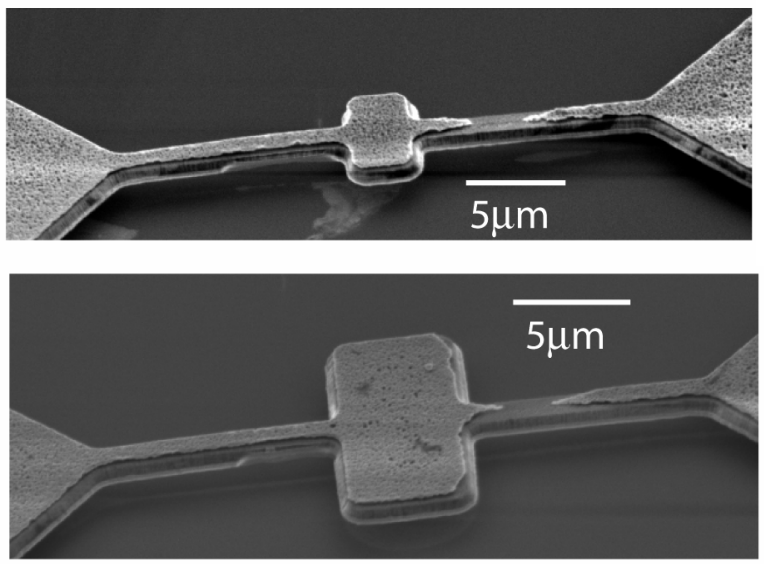

Figure 4. Scanning-electron micrographs of released resonator structures. TOP: $5 \mu \mathrm{m}$ long $x 3 \mu \mathrm{m}$ wide resonator. BOTTOM: $10 \mu \mathrm{m}$ long $\times 6 \mu \mathrm{m}$ wide resonator. Tethers are approximately $1 \mu \mathrm{m}$ wide for both devices.

\section{FABRICATION DETAILS}

CRITICAL ALIGNMENTS: The small device size requires precise alignments in the structure. For example, the resonator should be centered on the tethers to minimize sensing of nonlongitudinal modes, and the resonator and tethers should be centered on the support structure to allow minimization of acoustic wave propagation into the supports through tether-length "tuning." This process incorporates all of these alignments into the first mask level, which patterns the nickel. Since the nickel serves as an etch mask for the entire stack, the device definition is self-aligned. Additionally, the patterned device structure serves as a self-aligned etch mask for its own release.

MATERIAL/PROCESS CHOICES: Tradeoffs among device electrical characteristics, IC-compatible processing, and piezoelectric film growth requirements dictated materials selection.

Our AIN films were deposited by a commercial vendor [7]. Their best-quality process, which typically yielded an x-ray rocking curve FWHM of $\sim 1.5$ degrees, dictated the bottom of the piezoelectric stack (seed AlN/Mo/AlN). Nickel was chosen as the top metal for its suitability as an etch mask for both wet etching and Cl-based dry etching [8] of AlN, as well as for a ratio of density to electrical conductivity comparable to that of molybdenum.

The choice of molybdenum, aluminum nitride, and nickel required a unique set of selective etch chemistries, shown in Table 1.

\begin{tabular}{|c|c|c|c|c|c|}
\hline $\begin{array}{l}\text { rate/ } \\
\text { notes }\end{array}$ & $\begin{array}{c}\text { 20:1:1 } \\
\text { DI:HF: } \mathrm{H}_{2} \mathrm{O}_{2}\end{array}$ & $\begin{array}{c}\text { AZ400k } \\
\text { developer }\end{array}$ & $\begin{array}{c}\mathrm{CF}_{4} / \mathrm{O}_{2} \mathrm{RIE} \\
{[9]}\end{array}$ & $\mathrm{XeF}_{2}$ & BHF \\
\hline $\mathbf{N i}$ & $\sim 1000 \AA / \mathrm{min}$ & $\mathrm{x}$ & $\mathrm{x}$ & $\mathrm{x}$ & $\mathrm{x}$ \\
\hline AIN & $\mathrm{x}$ & $\begin{array}{c}\text { self- } \\
\text { limiting } \\
\text { (see text) }\end{array}$ & $\mathrm{x}$ & $\mathrm{x}$ & $\mathrm{x}$ \\
\hline Mo & $\mathrm{x}$ & $\mathrm{x}$ & $\begin{array}{l}\text { anisotropic } \\
\sim 133 \AA / \mathrm{min} \\
\end{array}$ & $\begin{array}{l}\text { isotropic, } \\
\text { undercut }\end{array}$ & $\mathrm{x}$ \\
\hline $\mathrm{SiO}_{2}$ & not exposed & $\mathrm{x}$ & not exposed & $\mathrm{x}$ & $\begin{array}{l}\text { re- } \\
\text { lease }\end{array}$ \\
\hline
\end{tabular}

Table1. Etch chemistries used to fabricate resonator. 
The Mo/AlN stack is deposited by sputtering/reactive sputtering at $400^{\circ} \mathrm{C}$ or below. No high-temperature steps are used in the subsequent processing of the device, making this process ICcompatible.
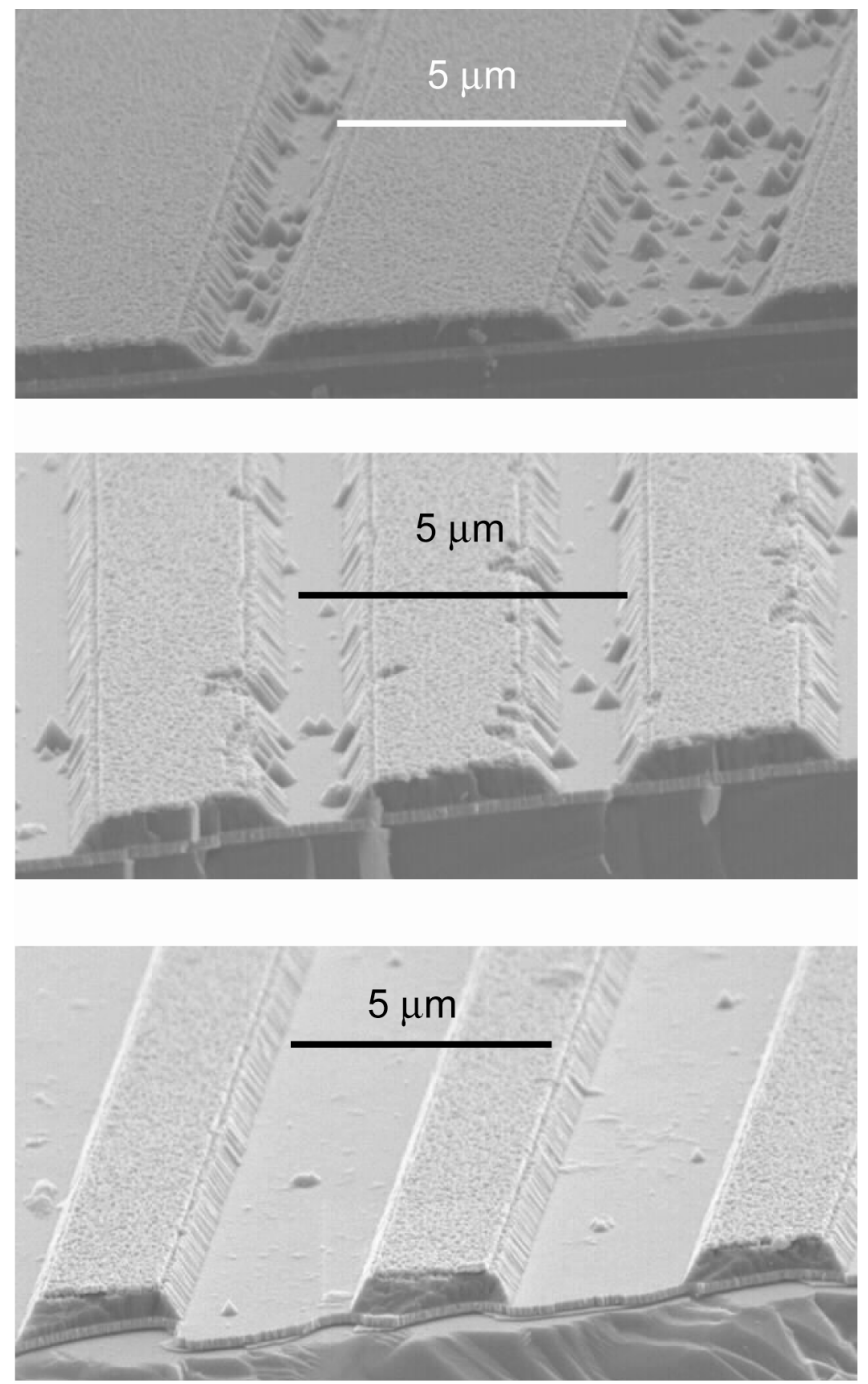

Figure 5. Scanning-electron micrograph of progress of AlN wet etch in AZ400k photoresist developer. The top Ni etch mask protects the underlying AlN film, which is on a Mo bottom layer. The surface of the exposed AlN quickly roughens into conicalshaped structures that slowly etch to the underlying Mo. TOP: 1:30 etch. MIDDLE: 2:30 etch. BOTTOM: 10:00 etch. The AlN etch is self-limited at the sidewall angle shown ( $\sim 50 x$ overetching showed no change in the structure).

WET ETCHING OF AlN: For our AlN films, wet etching in AZ400k photoresist developer was self-limiting at a fixed sidewall angle of approximately $55^{\circ}$. Figure 5 shows scanning-electron micrographs of a time sequence of the AIN etch. In unprotected regions, the etch produces conical structures. As the etch progresses, these cones shrink in size, eventually disappearing. Note that in the protected areas the film is dense, i.e. no etchant infiltrates from the sides.

The self-limiting nature of this etch is quite high; $\sim 50 \mathrm{x}$ overetching showed no change in the structure. Previous literature on etching of AlN in AZ400k does not mention film roughening, conical structures, or self-limited etching $[\mathbf{1 0}, \mathbf{1 1}]$. This effect is beneficial to our process, but not well understood at this time.

UNDERCUT ETCHING OF Mo: Minimizing stray capacitance required a break to be patterned in the top and bottom tether metal. For the top metal, this was a rather straightforward wet etch with a photoresist mask, but for the Mo bottom metal, an undercut etch was required.

To execute this etch, a resist window was opened in the area around the bottom metal break, and $\mathrm{XeF}_{2}$ etch $[12,13]$ was used to etch the $\mathrm{Mo} . \mathrm{XeF}_{2}$ gave high selectivity to all other materials in the structure, and was a highly isotropic etch.

\section{DEVICE MEASUREMENTS}

Resonator testing was conducted at the wafer level, in air, at room temperature, using GGB Industries probe tips on a Cascade Microtech probe station and an Agilent 8753 Network Analyzer. The device is wired in a series configuration for two-port measurements.
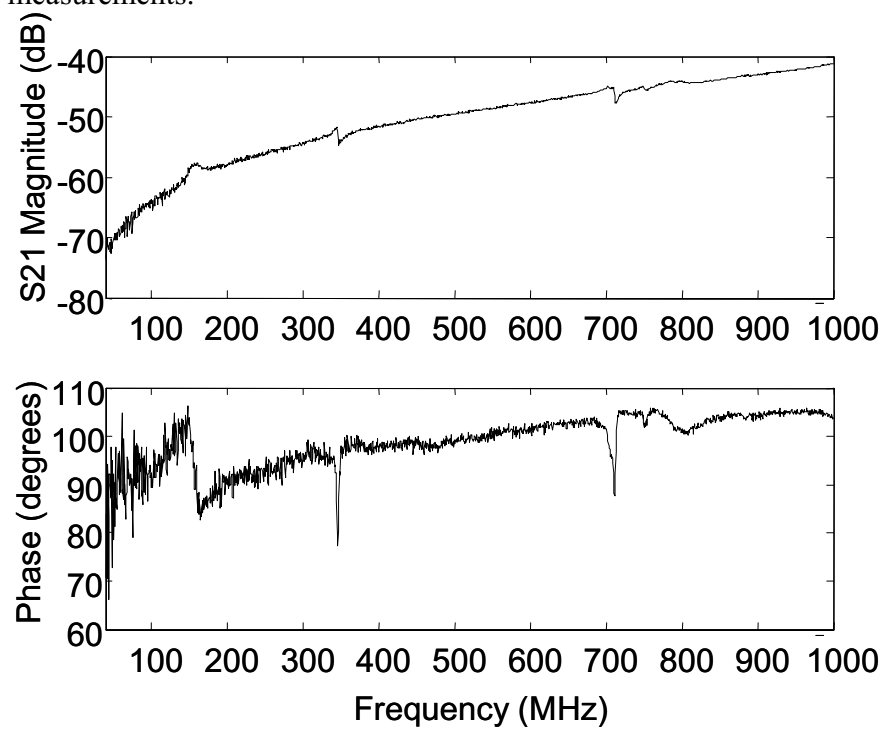

Figure 6. $S 21$ measurement of a 10- $\mu m x$ 5- $\mu m$ L-bar resonator. Two longitudinal-mode resonances are evident, corresponding to the length and width modes of the device.
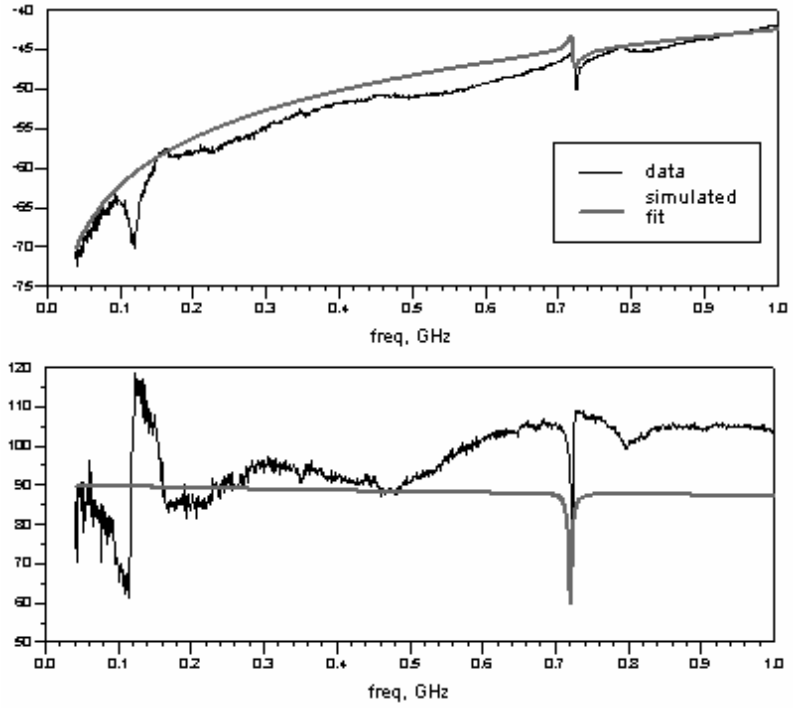

Figure 7. S21 data from a different 10- $\mu m \times 5$ - $\mu$ m device, along with fit to a BVD model. This model gives a $Q$ of 125. $Q$ values as high as 550 at $700 \mathrm{MHz}$ have been measured on these devices. 
Figure 6 shows an S21 measurement of a $10 \mu \mathrm{m}$ long x $5 \mu \mathrm{m}$ wide resonator over a wide frequency sweep. Two longitudinalmode resonances are evident, corresponding to the length and width modes, at approximately 350 and $700 \mathrm{MHz}$, respectively. The spurious feature at $120 \mathrm{MHz}$, which is currently not well understood, is present in all devices as well as non-resonant calibration structures.

Figure 7 shows a fit to a $5 \mu \mathrm{m}$ width mode from a different resonator. The fit uses a Butterworth van Dyke (BVD) model [14] for the mechanical resonance. Parasitics were modeled as port capacitance and resistance. The BVD circuit parameters were $\mathrm{R}=$ $35 \mathrm{k} \Omega, \mathrm{L}=1 \mathrm{mH}, \mathrm{C}=0.047 \mathrm{fF}$, and $\mathrm{Co}=2.7 \mathrm{fF}$. This corresponds to a $\mathrm{Q}$ of 135 . Q values as high as 550 at $700 \mathrm{MHz}$ have been measured on fabricated devices.
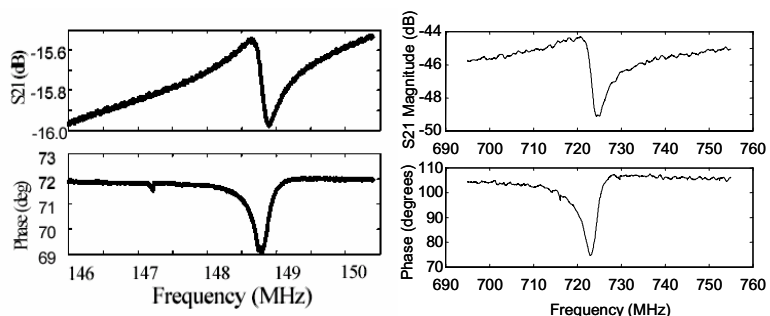

Figure 8. Closeups of S21 data from two other devices. LEFT: 25 um length mode. RIGHT: 5 um width mode.

Figure 8 shows detailed S21 data of resonances from two other devices. Figure 9 shows a plot of frequency vs. inverse length. The slope of $3.7 \mathrm{GHz}-\mu \mathrm{m}$ is consistent with calculations based on the AIN and metal layer mechanical properties for a simple longitudinal mode.

Frequency vs. Inverse Length

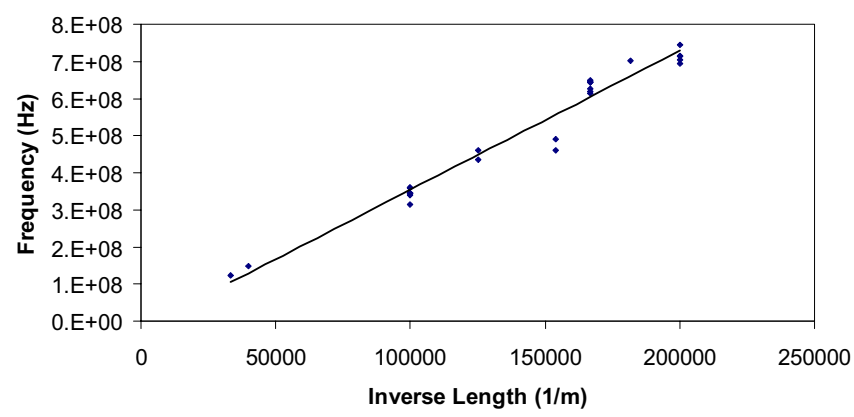

Figure 9. Frequency vs. inverse length for a number of resonators.

\section{CONCLUSIONS}

A new RF MEMS resonator has been designed, fabricated, and measured. The design addresses key performance parameters including quality factor, frequency scalability, mode isolation, and impedance matching. The design allows for a self-aligned fabrication process enabling high dimensional precision even as feature sizes are pushed to sub-micron ranges. A unique undercut etch is used to isolate the electrical leads and form the basic overlap capacitor structure. Initial measurements verify that wellisolated longitudinal modes are excited in the devices. Although measured quality factor and electrical admittance are much lower than theoretical limits predict, ongoing work will address electrical parasitics and process improvements. These first experimental results verify that the RF resonator design is widely scalable in frequency, confirm that the modes are well-isolated, and demonstrate an IC-compatible fabrication process.

\section{ACKNOWLEDGEMENTS}

The authors would like to thank the many Draper Laboratory staff and technicians who contributed to this work, particularly Connie Cardoso, Mert Prince, and Mark April for their work in the fabrication of the devices, Mark Mescher and Mathew Varghese for helpful discussions about many aspects of the work, and Jim Sitomer for program management support. This material is based upon work supported by the U.S. Army Aviation and Missile Command under Contract No. DAAH01-01-C-R204. Any opinions, findings and conclusions or recommendations expressed in this material are those of the authors and do not necessarily reflect the views of the Charles Stark Draper Laboratory, Inc.

\section{REFERENCES}

1. C.T.-C. Nguyen, "Micromechanical components for miniaturized low-power communications," Proceedings, 1999 IEEE MTT-S International Microwave Symposium RF MEMS Workshop, Anaheim, CA, June 18, 1999, pp. 48-77.

2. K.M. Lakin, "Thin Film Resonators and Filters," 1999 IEEE International Ultrasonics Symposium, Paper M-1, October 17-21, 1999.

3. M. Hara, J. Kuypers, T. Abe, M. Esashi, "MEMS Based Thin Film 2 GHz Resonator for CMOS Integration," IEEE MTT-S Digest, p. 1797, 2003.

4. R. Ruby, P. Bradley, Y. Oshmyansky, A. Chien, "Thin Film Bulk Wave Acoustic Resonators (FBAR) for Wireless Applications," IEEE Ultrasonics Symposium, p. 813, 2003.

5. D. T. Chang, et al., "A New MEMS-based Quartz Resonator Technology,” Hilton Head 2004.

6. J. Wang, Z. Ren, C.T.-C. Ngyuen, "Self-aligned 1.14-GHz vibrating radial-mode disk resonators," $12^{\text {th }}$ International Conference on Solid State Sensors, Actuators and Microsystems, Boston, June 8-12 2003.

7. Advanced Modular Sputtering (AMS), Goleta, CA, www.amsincorp.com.

8. A. N. Cleland, M. Pophristic, and I. Ferguson, "Single-crystal aluminum nitride nanomechanical resonators," Appl. Phys. Lett. 79(13), 2070 (2001).

9. Yue Kuo and J. R. Crowe, "Slope control of molybdenum lines etched with reactive ion etching," J. Vac. Sci. Technol. A 8(3), 1529 (1990).

10. J. R. Mileham et al., "Wet chemical etching of AlN," Appl. Phys. Lett. 67(8), 1119 (1995).

11. . J. R. Mileham et al., "Patterning of AlN, InN, and GaN in KOH-based solutions," J. Vac. Sci. Technol. A 14(3), 836 (1996).

12. Xactix,, Inc., Pittsburgh, PA, www.xactix.com.

13. Patrick B. Chu et al., "Controlled Pulse-Etching with Xenon Difluoride," Digest of Technical Papers, Volume 1, 1997

International Conference on Solid-State Sensors and Actuators (Transducers 97), Chicago, IL, pp. 665-668 (1997).

14. Lakin, K. M., "Modeling of thin film resonators and filters," IEEE MTT-S International Microwave Symposium Digest, Albuquerque, NM, pp. 149-152, June 1992. 